\section{Regards sur l'économie allemande}

Bulletin économique du CIRAC

109 | 2013

Varia

\title{
Union bancaire. La position des caisses d'épargne allemandes
}

\section{Georg Fahrenschon}

Traducteur : Isabelle Bourgeois

\section{OpenEdition \\ Journals}

Édition électronique

URL : http://journals.openedition.org/rea/4551

DOI : 10.4000/rea.4551

ISSN : 1965-0787

Éditeur

CIRAC

Édition imprimée

Date de publication : 4 juillet 2013

Pagination : 5-14

ISSN : 1156-8992

Référence électronique

Georg Fahrenschon, « Union bancaire. La position des caisses d'épargne allemandes », Regards sur l'économie allemande [En ligne], 109 | juillet 2013, mis en ligne le 01 juillet 2015, consulté le 01 mai 2019. URL : http://journals.openedition.org/rea/4551; DOI : 10.4000/rea.4551 


\section{Union bancaire. La position des caisses d'épargne allemandes}

\section{Georg Fahrenschon}

A en croire la presse française, l'Allemagne s'obstinerait à camper sur des positions « non constructives » lorsqu'il s'agit de renforcer l'Union économique et monétaire. « A chaque étape de la construction de l'Union bancaire, l'Allemagne voit toujours une raison de soulever des objections ", s'insurge par exemple le site euractiv.fr : « La supervision bancaire? Pas utile pour les petites banques. La recapitalisation directe? Pas possible avant une supervision effective des banques... La restructuration des banques par un nouvel organisme européen ? Pas avant un changement de traité " (18-04-2013)... Certes, la réalisation de I'Union bancaire est un dossier complexe, qui s'accompagne d'importants transferts de souveraineté, et se négocie à l'approche des élections européennes... Mais ce que révèle avant tout la guerre de tranchées menée contre l'Allemagne par les médias français, c'est l'existence de différences foncières d'approche entre nos deux pays - non pas sur le fond ni sur la finalité d'un tel projet européen - mais sur la manière de procéder à sa réalisation.

C'est là une différence classique entre nos deux pays, qu'il s'agisse de la culture en affaires ou en politique, et source intarissable de différends ; mais cette différence est aussi la justification même de cette obligation de résultat qu'a le " tandem ॥ franco-allemand dans l'Union. Et de fait, ce qui nourrit les griefs que véhicule le billet cité ci-dessus, c'est une "petite phrase " prononcée par Wolfgang Schäuble à l'issue de la réunion des ministres des Finances européens, le 13 avril. L'Union bancaire, expliquait-il, " n'a de sens que si nous avons des règles communes pour restructurer et faire face aux défaillances des banques. Si nous voulons des institutions européennes pour cela, il faut modifier le traité ». Le progrès des négociations depuis cette date n'a rien changé quant au fond de l'approche allemande: le politique doit se contenter de fixer un cadre pour l'activité des acteurs économiques, c'est-à-dire des règles contraignantes, dignes de confiance - donc fixées par voie de traité. Pour permettre aux lecteurs français de mieux comprendre les arguments sous-jacents de l'approche allemande, nous avons demandé à la Fédération des caisses d'épargne (DSGV), qui a joué un rôle éminent dans la définition des positions allemandes, de nous détailler ici dans leur complexité les différents arguments entrant en ligne de compte. (IB)

\section{Les étapes sur la voie d'une Union bancaire dans I'UE}

La crise de la finance mondiale, qui s'était déclenchée en 2007 et dont l'effondrement de la banque d'investissement Lehmann Brothers en septembre 2008 avait marqué le point culminant, a révélé au grand jour que, pratiquement dans le monde entier, la gestion de crise et la gestion des liquidités des banques présentent des faiblesses parfois considérables. En réaction à ce constat, lors de leur sommet à Pittsburgh à la fin 2008, les membres du G20 se sont rapidement mis d'accord pour que, à l'avenir, les banques couvrent leurs risques avec un volume plus élevé de capitaux, et de capitaux de meilleure qualité. Parallèlement, ils ont décidé d'adopter une réglementation mondiale pour les liquidités bancaires afin d'assurer que les établissements disposent d'une couverture financière suffisante garantissant leur solvabilité à court comme à long terme.

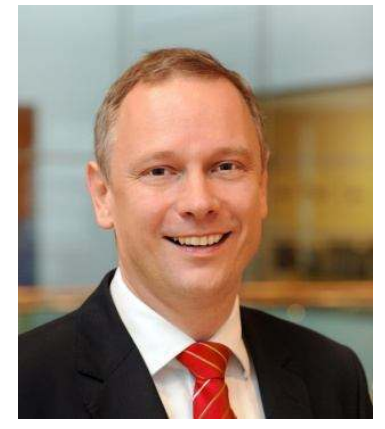

Georg Fahrenschon, Président de la Fédération allemande des caisses d'épargne

Deutscher Sparkassen- und Giroverband (DSGV)
Vers une réglementation mondiale pour les liquidités bancaires 
Vers une surveillance plus efficiente dans l'UE

Un MUS pour rompre le lien entre risques souverains et risques bancaires...

... sans mettre à contribution les contribuables de l'UE

Commission : " Feuille de route pour une union bancaire »
Mais lors de la crise sont apparus d'autres dysfonctionnements encore, révélant que les autorités de régulation et de surveillance portent leur part de responsabilité aussi bien dans l'origine de la crise que dans l'ampleur qu'elle a prise. Car dans de nombreux domaines, il aurait fallu identifier ces dysfonctionnements plus tôt et prendre les mesures s'imposant pour les enrayer. Encore fallait-il que ce soit réalisable, tout particulièrement dans le cas d'établissements financiers dont l'activité ne s'arrête pas aux frontières. Or, à de nombreuses reprises, les autorités de surveillance avaient invoqué des "zones blanches " leur interdisant de collecter par-delà les frontières nationales les informations et données indispensables pour effectuer une évaluation exhaustive des risques pesant sur une banque ou un groupe d'établissements.

La réponse fut un appel à l'extension des compétences en matière de surveillance et à la création de nouvelles structures afférentes. Au sein de l'UE fut ainsi adopté dès novembre 2010 un règlement instituant des autorités européennes de surveillance pour les secteurs de la banque, de l'assurance et des marchés de valeurs mobilières. Et avec l'institution à Londres, au début de 2011, de l'Autorité bancaire européenne (ABE), naquit la première autorité de supervision agissant par-delà les frontières. Ses objectif et mission étaient notamment de contribuer à I'harmonisation des pratiques de supervision au sein de l'UE en procédant à l'uniformisation des normes et pratiques prudentielles et en assurant une meilleure coordination entre les autorités de surveillance des Etats membres. Quant aux autorités européennes de surveillance (assurances, marchés financiers, risque systémique...), créées elles aussi en 2010 pour assurer la coordination de la supervision des groupes d'établissements financiers transfrontaliers, elles avaient pour mission de développer l'échange d'informations et de renforcer la coordination des mesures de surveillance.

Mais au plus tard après les difficultés (au demeurant prévisibles) rencontrées par I'ABE dès sa prise de fonction, et qui se sont clairement manifestées lors de la mise en œuvre des tests de résistance bancaire ("stress tests »), force fut de constater que ces outils ne suffisaient pas à enrayer l'évolution de la crise de la finance mondiale. De plus, avec l'extension de la crise de la dette souveraine au sein de la zone Euro, les Etats et les banques furent confrontés à de nouveaux défis. Afin de couper le lien d'interaction entre hausse de l'endettement et crises à répétition du système bancaire, les Etats membres de la zone Euro décidèrent alors en juin 2012 que les établissements financiers en difficulté auraient accès directement aux fonds du Mécanisme européen de stabilité (MES), c'est-à-dire sans passer par l'intermédiaire des budgets des Etats. Toutefois, cette possibilité était d'emblée assortie d'une condition: les banques ne pourront se recapitaliser directement auprès du MES qu'après la mise en place effective d'un Mécanisme unique de supervision (MUS), c'est-à-dire grâce au transfert de missions de supervision à la Banque centrale européenne selon l'art. $127 \S 6$ du Traité sur le fonctionnement de l'UE (TFUE).

Cette disposition montre que le motif décisif menant à la création du Mécanisme unique de supervision n'était pas réellement la préoccupation d'instituer une supervision efficiente du système bancaire, et tout particulièrement des établissements transfrontaliers. Sa création répondait plutôt à la volonté - compréhensible au demeurant - de trouver une solution à une situation peu acceptable : à savoir que les établissements bancaires se trouvant en crise entre autres du fait de défaillances de la surveillance à l'échelon national aient la possibilité de se recapitaliser directement auprès de fonds européens alimentés par les contribuables de I'UE, alors qu'ils restent soumis à la seule surveillance des instances nationales. Dans ce cas précis en effet, une certaine congruence entre le recours à des fonds européens et une surveillance européenne s'impose à l'évidence.

Après que les chefs d'Etat et de gouvernement de la zone Euro eurent, en conséquence, confié à la Commission le mandat d'élaborer des propositions pour l'institution d'un Mécanisme unique de supervision, celle-ci rendit en septembre 2012 une "Feuille de route pour une union bancaire ». Cet important paquet de me- 
sures ne porte pas seulement sur le projet pour lequel elle avait été mandatée le mécanisme de surveillance à instituer sous l'égide de la BCE -, mais préconise en outre une gestion intégrée des crises bancaires, combinée à un mécanisme de résolution unique compétent dans la mesure du possible pour l'ensemble de I'Union, et à un système européen de protection des dépôts. Or en formulant ces préconisations, la Commission outrepasse largement les objectifs pour lesquels elle avait été mandatée par les Etats membres de la zone Euro. En particulier, la préconisation de créer un système unique, européen, de protection des dépôts ne figurait nullement parmi les recommandations que lui avaient demandé de formuler les chefs d'Etat et de gouvernement. De plus, un tel système aurait présenté le risque d'insécuriser encore plus les épargnants dans ces Etats de la zone Euro remplissant les conditions de stabilité, car elle aurait nourri leurs craintes de voir que leur système de garantie des dépôts pourrait s'appliquer aux épargnants des pays en crise. Le cas de Chypre (dans leur première proposition, les ministres des Finances de l'Eurogroupe avaient prévu que même les épargnants chypriotes protégés par la garantie des dépôts, c'est-à-dire ceux dont les placements étaient inférieurs à $100000 €$, devaient renoncer à une partie de leur épargne) nous a montré quelles peuvent être les conséquences d'un système unique, européen, de garantie des dépôts pour les systèmes de garanties des autres Etats.

En décembre 2012, les chefs d'Etat et de gouvernement de l'UE ont alors adopté une contre-proposition aux préconisations de la Commission : rejetant l'idée première d'une supervision unique et directe de tous les établissements bancaires confiée à la $\mathrm{BCE}$, ils ont décidé une sorte de répartition des tâches entre la banque centrale européenne et les autorités nationales de surveillance. Le critère déterminant adopté dans ce cadre est le caractère "significatif » (systémique) des établissements soumis à la supervision prudentielle.

Par cette décision, le Conseil a répondu notamment à une demande de l'Allemagne qui refusait l'idée que la supervision par la BCE s'applique sans distinction à toutes les banques de la zone Euro et considérait à l'inverse que seules les autorités de surveillance nationales, fortes de leur "proximité avec le marché », peuvent être compétentes pour les petites banques régionales ou celles dont le rayon d'action se cantonne à leur commune d'implantation. Cette position visait à mieux prendre en considération les particularités du système bancaire allemand avec ses trois piliers que sont les banques commerciales, les banques coopératives et les établissements de droit public (les Sparkassen appartiennent à cette dernière catégorie), ainsi que, plus généralement, son architecture polycentrique. De plus, une telle répartition des rôles concorde avec le principe de subsidiarité qui joue un rôle non négligeable tout particulièrement en ce qui concerne le partage des missions et compétences au sein de l'Union.

Sont considérés comme " significatifs » (" significant ») les établissements financiers dont les actifs dépassent les 30 milliards $€$ ou pèsent plus de $20 \%$ du PIB de l'Etat membre concerné. Par ailleurs, la BCE peut étendre sa supervision aux établissements ayant des activités transfrontières (par exemple des filiales à l'étranger). Enfin, la banque centrale disposera d'un droit d'intervention lui permettant également d'assurer la surveillance directe de banques ne répondant pas à ce critère systémique, dès lors qu'elle estime que cela est nécessaire pour garantir l'application uniforme des normes prudentielles ou lorsque ces banques bénéficient d'aides directes du FESF ou du MES ou en font la demande. Le Mécanisme unique de supervision vaut automatiquement pour les 17 Etats membres de la zone Euro. Les Etats de l'UE qui ne font pas partie de la zone Euro peuvent choisir librement de prendre part à ce mécanisme en engageant avec la BCE une " coopération étroite » en qualité d'Etats membres participants.

La procédure de trilogue, engagée le 18 décembre 2012 sur la négociation, par le Conseil des ministres de l'UE, le Parlement européen et la Commission, d'un accord sur un règlement confiant à la BCE des pouvoirs spécifiques en vue de la supervision des établissements financiers (règlement instituant le MUS), et
Contre-proposition des chefs d'Etat et de gouvernement de I'UE : ...

... respect du principe de subsidiarité

Définition de banques « significatives »

Le trilogue aboutit à un compromis sur plus de transparence démocratique 
d'autre part sur un amendement du règlement instituant une Autorité bancaire européenne (l'ABE, installée à Londres, fixe les normes et règles auxquelles sont soumises toutes les banques de l'UE), a abouti le 19 mars 2013 à un compromis politique entre les parties concernées. Celui-ci prévoit pour l'essentiel le renforcement du contrôle démocratique de l'autorité de supervision par le Parlement européen (approbation du président et du vice-président du conseil de surveillance du conseil en charge de la supervision à instaurer auprès de la BCE (Board of Supervisors), le renforcement du contrôle parlementaire, en partie par les parlements nationaux, de la politique de surveillance menée par la BCE, de même qu'une stricte séparation entre les tâches de supervision de la BCE et ses fonctions liées à la politique monétaire, ainsi que la séparation organisationnelle des personnes en charge de ces missions. En conséquence de quoi, lors du vote de ces propositions en séance plénière, le 22 mai 2013, le Parlement européen a soumis son accord final à une condition sine qua non : l'engagement formel et contraignant de la BCE, fixé par traité, à rendre compte devant le Parlement européen - ceci afin que la BCE ne puisse pas se prévaloir, en matière de supervision, de l'indépendance que lui garantissent par définition les traités de l'UE.

\begin{tabular}{|c|c|}
\hline \multicolumn{2}{|c|}{ Bref aperçu de l'état de la réglementation relative à l'Union bancaire } \\
\hline Textes & Etat au $1^{e r}$ juillet 2013 \\
\hline $\begin{array}{l}\text { Proposition de règlement du Parlement européen et du Conseil concernant les exigences } \\
\text { prudentielles applicables aux établissements de crédit et aux entreprises d'investissement } \\
(\text { COM(2011)0452-C7-0417/2011-2011/0202 (COD)) }\end{array}$ & $\begin{array}{l}\text { Publication au Journal officiel de l’UE le } 27 \\
\text { juin } 2013 \text {; } \\
\text { entrée en vigueur le } 1^{\text {er }} \text { janvier } 2014\end{array}$ \\
\hline $\begin{array}{l}\text { Proposition de directive du Parlement européen et du Conseil concernant l'accès à l'activi- } \\
\text { té des établissements de crédit et la surveillance prudentielle des établissements de crédit } \\
\text { et des entreprises d'investissement, modifiant la directive } 2002 / 87 / \text { CE du Parlement euro- } \\
\text { péen et du Conseil relative à la surveillance complémentaire des établissements de crédit, } \\
\text { des entreprises d'assurance et des entreprises d'investissement appartenant à un con- } \\
\text { glomérat financier (COM (2011)0453 - C7-0210/2011 - 2011/ 0203(COD)) }\end{array}$ & $\begin{array}{l}\text { Publication au Journal officiel de l'UE le } 27 \\
\text { juin } 2013 \text {; } \\
\text { entrée en vigueur le } 1^{\text {er }} \text { janvier } 2014\end{array}$ \\
\hline $\begin{array}{l}\text { Proposition de règlement du Conseil confiant à la Banque centrale européenne des mis- } \\
\text { sions spécifiques ayant trait aux politiques en matière de contrôle prudentiel des établis- } \\
\text { sements de crédit (COM(2012) } 511-2012 / 0242 \text { (CNS)) }\end{array}$ & $\begin{array}{l}\text { Adoption finale imminente par le Parlement } \\
\text { européen ; la BCE devrait exercer ses mis- } \\
\text { sions de supervision dès septembre } 2014\end{array}$ \\
\hline $\begin{array}{l}\text { Proposition de règlement modifiant le règlement (UE) } n^{\circ} 1093 / 2010 \text { instituant une Autorité } \\
\text { européenne de surveillance (Autorité bancaire européenne) (COM(2012) } 512-2012 / 0244 \\
(\text { COD)) }\end{array}$ & $\begin{array}{l}\text { Ce règlement sera adopté en même temps } \\
\text { que le règlement confiant à la BCE des } \\
\text { missions spécifiques }\end{array}$ \\
\hline $\begin{array}{l}\text { Communication de la Commission au Parlement européen et au Conseil « Feuille de route } \\
\text { pour une union bancaire » }(\operatorname{COM}(2012) 510)\end{array}$ & $\begin{array}{l}\text { Publication au Journal officiel de l'UE le } 12 \\
\text { septembre } 2012 \text {; prochaines étapes pré- } \\
\text { vues mi-juillet } 2013\end{array}$ \\
\hline $\begin{array}{l}\text { Proposition de directive du Parlement européen et du Conseil établissant un cadre pour le } \\
\text { redressement et la résolution des défaillances d'établissements de crédit et d'entreprises } \\
\text { d'investissement (COM/2012/0280 - 2012/0150 (COD)) }\end{array}$ & $\begin{array}{l}\text { Le Conseil a adopté sa position le } 27 \text { juin } \\
2013 \text {; négociations au sein du trilogue sur } \\
\text { la base de cette position }\end{array}$ \\
\hline $\begin{array}{l}\text { Proposition de directive du Parlement européen et du Conseil modifiant la directive 94/19/ } \\
\text { CE relative aux systèmes de garantie des dépôts en ce qui concerne le niveau de garantie } \\
\text { et le délai de remboursement (COM(2008)0661 - C6-0361/2008 - 2008/ 0199(COD)) }\end{array}$ & $\begin{array}{l}\text { Adoption imminente par le Parlement euro- } \\
\text { péen et le Conseil ; entrée en vigueur } \\
\text { prévue le } 1^{\text {er }} \text { janvier } 2014\end{array}$ \\
\hline
\end{tabular}

A moyen terme, modification du traité TFUE pour mieux asseoir le MUS

En cours : garantie des dépôts et résolution bancaire
Le Conseil des ministres de l'UE et la Commission ont choisi d'asseoir ce règlement relatif au Mécanisme unique de supervision (MUS) sur l'art. $127 \S 6$ du Traité sur le fonctionnement de l'UE (TFUE). Celui-ci stipule que «le Conseil, statuant par voie de règlements conformément à une procédure législative spéciale, à l'unanimité, et après consultation du Parlement européen et de la Banque centrale européenne, peut confier à la Banque centrale européenne des missions spécifiques ayant trait aux politiques en matière de contrôle prudentiel des établissements de crédit et autres établissements financiers, à l'exception des entreprises d'assurances ». La majorité des membres du Conseil considère le recours à l'art. $127 \S 6$ du TFUE comme une mesure satisfaisante dans un premier temps pour instituer le MUS. Pour sa part, le gouvernement fédéral, qui a toujours exprimé ses doutes quant à la solidité de cette base juridique, a insisté pour que soit mentionné, dans la déclaration finale, qu'une modification du traité est conjointement envisagée à moyen terme.

Ensuite, une fois ce MUS mis en place, un accord devait être trouvé d'ici le Conseil de la fin juin 2013 sur deux projets de directives: l'une portant sur le redressement des banques et la résolution de leurs défaillances (" gestion de 
crise »), l'autre sur la garantie des dépôts. Par ailleurs, lors du Conseil européen des 13 et 14 décembre 2012, les Etats membres avaient décidé que la Commission présentera, dans le courant 2013, une proposition relative à un mécanisme de résolution unique pour les Etats membres participant au MUS. Celui-ci devra s'appuyer sur les contributions du secteur financier afin de préserver les contribuables. Concrètement, cela signifie que le soutien public apporté le cas échéant à un dispositif de résolution sera refinancé grâce à un prélèvement a posteriori sur le secteur bancaire. Le 27 juin 2013, le Conseil est parvenu à un accord final sur un mécanisme de redressement et de résolution bancaire et a fixé, dans le projet de directive afférent, l'ordre de la répartition des pertes et garanties à mettre en œuvre entre les créanciers dans ce cadre. Ainsi, pour la première fois seront mis à contribution lors de la liquidation d'une banque les gros déposants, c'est-à-dire ceux dont les comptes dépassent $100000 €$.

\section{La supervision doit reposer sur des bases juridiques solides}

La décision de transférer, au sein d'un Mécanisme unique de supervision, les compétences en matière de supervision à la Banque centrale européenne marque l'étape décisive dans la mise en œuvre de la Feuille de route pour une Union bancaire européenne présentée par la Commission en septembre 2012. Dans ce contexte, les caisses d'épargne allemandes saluent la clarté de la délimitation des établissements placés sous la supervision de la BCE qui se traduit par le fait que les caisses d'épargne allemandes - à l'exception de la Sparkasse de Hambourg (et probablement aussi de celle de Berlin) - restent sous la supervision des autorités nationales (BaFin et Bundesbank). Nous considérons cette délimitation, négociée en grande partie à l'initiative de la partie allemande tant au sein du Conseil qu'au Parlement européen, comme un succès. Néanmoins, nous considérons que le seuil de 30 milliards $€$ d'actifs est trop bas et nous revendiquons une lecture dynamique de ce critère pour garantir d'une part que les établissements dont les actifs oscillent autour de ce seuil de 30 milliards $€$ ne soient pas soumis, au gré des fluctuations de leur bilan, à la permanente variation des autorités compétentes, et d'autre part pour exclure que, même dans le cas dune croissance organique du total des actifs, et dès lors que celle-ci n'est pas significative au point de faire entrer l'établissement en question dans la catégorie des banques systémiques, cette banque passe alors automatiquement dans le périmètre de la supervision par la BCE.

Les Sparkassen allemandes ont en effet toujours souligné l'importance - surtout pour des établissements dont les activités se cantonnent au marché national - à accorder à une grande proximité avec le marché comme à la connaissance intime de ce marché qui en résulte comme des éléments essentiels sur lesquels doit se fonder le futur Mécanisme unique de supervision. Certes, les règlements en cours de négociation confient en dernier ressort à la BCE la responsabilité de la supervision de tous les établissements de crédit, mais elle n'est pas habilitée pour autant à émettre des consignes particulières aux établissements ne présentant pas une taille significative. II en va autrement des conglomérats financiers et des autres catégories d'établissements: dans ce cas, la BCE peut émettre des consignes générales ("general instructions "; du même type par exemple que les minima requis en matière de gestion des risques - MaRisk - tels qu'ils s'appliquent aux banques allemandes). Le non-respect éventuel de ces consignes générales peut justifier le droit d'intervention de la BCE. D'une manière plus générale, une disposition tout particulièrement suscite notre inquiétude : que la BCE décide, du moins pour les établissements placés sous sa supervision directe, d'imposer les normes comptables IFRS et qu'elle se base sur ces mêmes normes prudentielles pour la supervision. Nous redoutons en outre qu'une telle décision enclenche une application uniforme de ces normes et accroisse la pression sur les établissements sous supervision nationale pour qu'ils adoptent eux aussi le standard IFRS dans le but de faciliter la comparabilité des bilans. Pour la plupart des banques allemandes, cela impliquerait en effet de lourds ef-
Délimitation efficiente des banques placées sous la supervision de la BCE

Les standards IFRS sont contraires au modèle économique des Sparkassen 
Confier la supervision à la $\mathrm{BCE}$ représente un important transfert de souveraineté

II requiert une révision des traités,...

... une stricte séparation des pouvoirs au sein de la BCE...

... et un droit administratif européen spécifique forts pour passer des normes comptables allemandes, régies par le Code du commerce (Handelsgesetzbuch) et assises sur le principe de prudence, aux standards internationaux axés en priorité sur la juste valeur et qui visent plutôt à satisfaire les exigences de transparence des investisseurs et des marchés financiers. Une telle approche est en outre totalement étrangère au modèle économique typique d'une Sparkasse allemande.

Le transfert des compétences en matière de supervision à la BCE est une avancée majeure vers la construction d'un système de supervision financière dans I'UE. Pour la première fois en effet, après l'institution en 2010 dans l'UE d'autorités nouvelles pour la surveillance bancaire (Autorité bancaire européenne, ABE), la surveillance des marchés financiers (European Securities and Markets Authority, ESMA) et celle des assurances, sera mis en place un Mécanisme unique de supervision qui, grâce au règlement accordant des pouvoirs étendus à la BCE, opérera un transfert de compétences en matière de supervision à une instance européenne. La portée, en droit constitutionnel, d'un tel transfert de souveraineté à l'échelon européen se comprend mieux quand on sait qu'il requiert, de l'avis du gouvernement fédéral, le vote d'une loi d'approbation en application de l'art. $23, \S$ 1, 1 de la Loi fondamentale. Cela étant, même une approbation par le Bundestag et le Bundesrat ne pourra pas remédier à un transfert de compétences à la BCE a priori contraire aux traités européens. Car - et c'est l'opinion générale - dans I'hypothèse où ce transfert serait conforme au droit, aucune loi d'approbation ne serait requise. Mais dans l'hypothèse où ce transfert serait contraire à la Constitution, le législateur ne serait en aucune manière habilité à adopter une loi d'approbation afférente dans le cas où on la soumettrait à son vote.

Un transfert de compétences de cette ampleur, impliquant d'importants pouvoirs d'intervention, présuppose donc que, en droit européen aussi, le MUS repose sur des bases juridiques stables et solides. Or du fait de l'urgence accordée à ce dossier à l'échelon communautaire, les bases juridiques sur lesquelles repose le fonctionnement du MUS ne sont actuellement pas assez solides. L'art. 127, $\S 6$ du Traité TFUE autorise certes que soient confiées à la BCE, sous les conditions décrites, des missions "spécifiques » ayant trait aux politiques en matière de contrôle prudentiel des établissements de crédit et autres établissements financiers. Mais le projet de règlement relatif au MUS prévoit à l'inverse de confier entièrement à la BCE des missions de surveillance clés pour la supervision d'une partie du système bancaire européen (comme l'agrément ou la fermeture d'établissements, la supervision de la solvabilité et de la liquidité). S'ajoute à cela que le projet de règlement prévoit expressément de déléguer en totalité à la BCE la responsabilité en ce qui concerne le fonctionnement du MUS. Or les autorités de surveillance nationales sont soumises aux directives de la BCE. Ce transfert de compétences dépasse de loin l'état de fait régi par l'art. 127, § 6 du Traité TFUE et exige à notre sens une modification des traités à l'échelon européen.

Nous attendons du gouvernement fédéral qu'il s'engage, au sein du Conseil de I'UE, pour que soient, sans tarder, modifiés les traités. Cela concorde au demeurant avec les conclusions du Conseil informel de l'ECOFIN les 12 et 13 avril 2013, au cours duquel a été adoptée une "déclaration politique » en ce sens : le droit européen doit être révisé de manière à ce que l'architecture de la supervision sous l'égide de la BCE soit adaptée à une stricte séparation des tâches et missions de la BCE. Ce n'est qu'ainsi que l'action du Mécanisme unique de supervision européen pourra être assise sur des fondements clairs et solides.

En outre, les textes en négociation présentent des lacunes en ce qui concerne les dispositions applicables aux procédures administratives relatives au rôle de la $\mathrm{BCE}$. Etant donné que les dispositions sur la supervision prévues par le règlement ont un lourd impact sur les droits fondamentaux (ils sont de nature à enfreindre aussi bien la garantie du droit de propriété que celle de la liberté d'exercer une profession), il convient d'adopter, pour la procédure des décisions et actes administratifs de la BCE comme l'ordonnance de fermeture d'une banque, un droit spécifique régissant les procédures administratives et garantissant une 
protection juridique efficiente. Un tel droit administratif européen spécifique existe d'ores et déjà en matière de droit communautaire de la concurrence ou de législation européenne sur les aides d'Etat.

Le choix de la BCE comme autorité en charge de la supervision directe des banques systémiques ( significatives ») soulève en outre la question de la gestion d'un éventuel conflit d'intérêts au sein de la BCE entre ses compétences en matière de politique monétaire et celles de supervision. De l'avis unanime des banques allemandes, il convient d'établir une plus stricte séparation des tâches se répercutant en particulier sur les compétences et instances décisionnelles respectives au sein de la BCE. La structure organique et décisionnelle prévue par la proposition de règlement confiant à la $\mathrm{BCE}$ des missions spécifiques en matière de contrôle prudentiel prévoit une sorte de " droit de veto " du Conseil des gouverneurs contre les projets de décisions du comité de surveillance. Par ailleurs, il incombe au Conseil des gouverneurs d'édicter des dispositions internes réglant ses relations avec le comité de surveillance. Or ces dispositions ne suffisent pas à garantir une séparation non équivoque entre les différentes missions confiées à la BCE, ce qui laisse présager des conflits d'intérêts.

\section{L'architecture du futur mécanisme européen de surveillance unique}

$\underline{\mathrm{UE} 17}$ (Euro) + X:

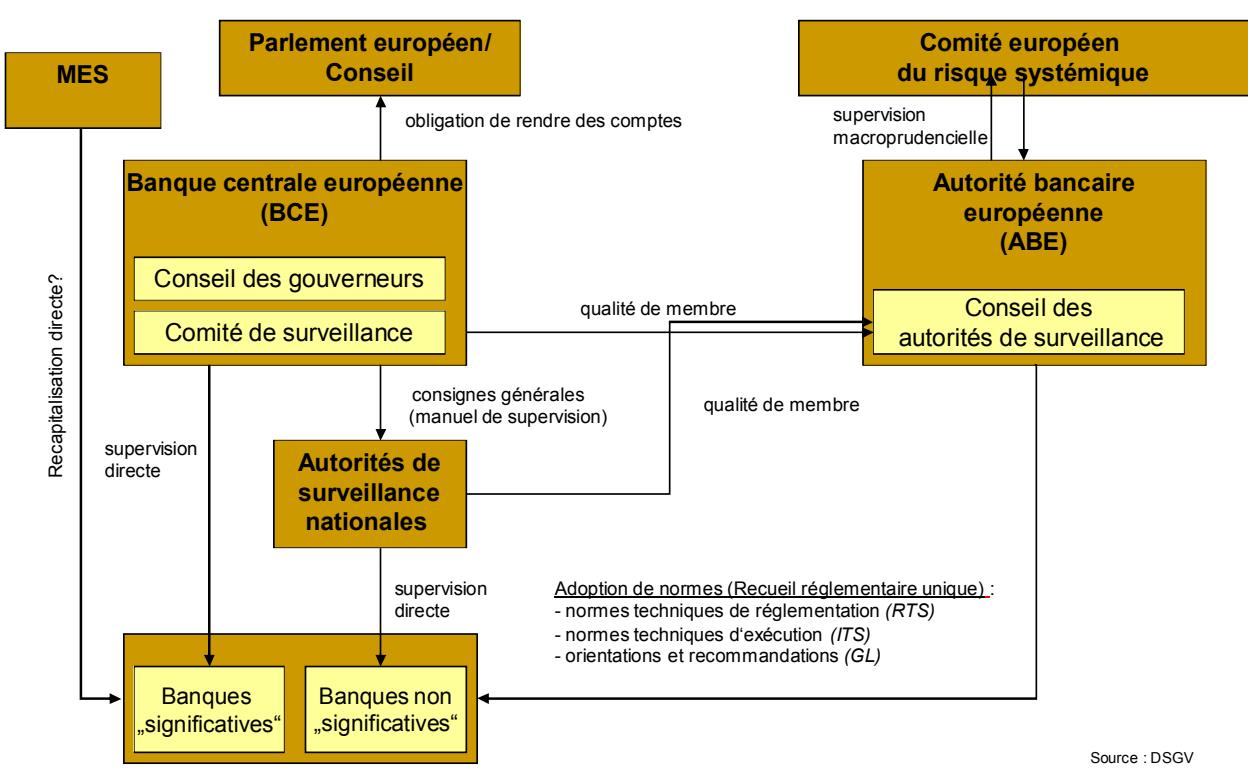

Adapter en conséquence la gouvernance de la BCE

L'organisation et le fonctionnement du nouveau mécanisme de supervision doivent également avoir pour objectif de réduire les doublons et d'éviter que les établissements de crédit placés sous sa surveillance subissent une surcharge bureaucratique. Cela nécessite de clarifier la délimitation des compétences respectives entre la BCE, l'Autorité bancaire européenne et les autorités de surveillance nationales. De l'avis des banques allemandes, il convient donc d'arrêter des dispositions contraignantes déterminant quelle autorité est compétente pour quelles tâches en matière de supervision et de quels pouvoirs administratifs elle dispose à cet effet, définissant comment doivent être exécutés les tests de résistance bancaire, et comment doivent être coordonnées les mesures de stabilisation financière en cas de défaillance bancaire (gestion de crise). Cela implique également de réduire les doublons en veillant à ce que les informations requises ne soient pas transmises inutilement aussi bien à l'autorité nationale qu'à la BCE (notamment concernant les critères de liquidité et de solvabilité), ainsi que toutes données générales ou spécifiques dont souvent les autorités demandent la

Clarifier l'articulation entre autorités nationales et européennes... 
... le droit d'intervention de la BCE...
France et Allemagne opposées à la centralisation

Le MES ne devrait intervenir qu'en dernier ressort... transmission ad hoc. II conviendrait donc d'établir, dans le futur règlement confiant les missions de contrôle prudentiel à la $\mathrm{BCE}$, des règles claires relatives à la circulation de l'information pour la supervision des établissements de crédit par les autorités nationales et pour la coopération avec ces dernières. Cela permettrait aux autorités nationales de surveillance de soumettre les données fournies à un contrôle de qualité avant de les transmettre à la BCE.

II conviendrait également de fixer des règles plus claires et plus prévisibles concernant le droit d'intervention de la BCE lui permettant dans certains cas d'assurer la surveillance directe des établissements de crédit n'entrant pas dans la catégorie des banques "significatives ». La disposition afférente du projet de règlement confiant les missions de contrôle prudentiel à la BCE se contente de stipuler que cette surveillance vise à garantir l'application uniforme de normes prudentielles ambitieuses ; elle manque de précision et de netteté.

Il convient de même de lever l'ambiguïté de la répartition des tâches entre la fixation de normes prudentielles et la surveillance au sein du Mécanisme uique de supervision. Elle présente en effet le risque de générer, de la part des différentes autorités de régulation et de supervision, une mosaïque d'exigences divergentes nuisible à une application cohérente des règles et susceptible de vider de sa substance les compétences de l'Autorité bancaire européenne en matière de fixation de normes prudentielles. Et ce risque est manifeste du fait des pouvoirs conférés à la $\mathrm{BCE}$ d'édicter ordonnances, directives et consignes générales tant envers les autorités nationales qu'envers les groupes ou conglomérats d'établissements de crédit bancaires et, d'autre part, de réaliser un manuel de supervision (" supervisory handbook ») unifié.

\section{Résolution : mise en réseau plutôt qu'une autorité centrale}

Le 30 mai 2013, dans une déclaration commune intitulée "La France et l'Allemagne ensemble pour renforcer l'Europe de la stabilité et de la croissance ", et publiée dans le prolongement des annonces faites le 22 janvier 2013 à l'occasion du $50^{\mathrm{e}}$ anniversaire du Traité de l'Elysée, la France et l'Allemagne ont apporté une nouvelle pierre à l'édifice. Cette déclaration précise en effet clairement que, parallèlement à la mise en œuvre du Mécanisme de srveillance unique, la directive sur le redressement des banques et la résolution de leurs défaillances et la directive sur la garantie des dépôts " devront avoir été conclues par le Conseil d'ici à la fin juin 2013 puis approuvées par le Parlement européen ». De même, "les éléments essentiels régissant les critères opérationnels pour la recapitalisation directe des banques devront avoir été élaborés d'ici à la fin juin 2013 », parallèlement aux négociations relatives à ces deux directives.

C'est sur cette base que devra s'effectuer la mise en place d'un mécanisme de résolution unique - en premier lieu pour les Etats membres de la zone Euro comme pour les Etats participant en outre au MUS. L'un des principes clés évoqués dans cette déclaration est celui d'un " conseil de résolution unique associant les autorités de résolution nationales et permettant une prise de décision rapide, efficace et cohérente au niveau central ". En l'absence de traités européens régissant l'institution d'un mécanisme de résolution unique, la France et l'Allemagne prennent position contre l'institution d'un mécanisme de résolution européen tel que le prône la Commission et plaident à l'inverse pour la mise en réseau des autorités de résolution nationales.

Les autres principes clés figurant dans la déclaration sont que « le mécanisme de résolution unique devrait s'appuyer sur les contributions du secteur financier luimême, assurant à terme le préfinancement d'un dispositif de soutien privé approprié et effectif ». Le soutien du MES ne devrait être invoqué qu'en ultime ressort, qu'il s'agisse d'accorder un crédit aux Etats membres ou de recapitaliser directement des établissements bancaires. Dans ce cadre, la France et l'Allemagne pourraient étudier la possibilité de rapprocher ultérieurement le mécanisme de résolution unique (MRU) et le MES. L'accord auquel est parvenu le Conseil euro- 
péen le 27 juin 2013 prévoit clairement le recours aux fonds nationaux de résolution bancaire. Néanmoins, la Commission a annoncé parallèlement qu'elle présenterait, à l'été 2013, une recommandation en vue de l'institution d'un MRU comprenant un fonds de résolution unique et une autorité unique.

II est déterminant à nos yeux que le déroulement concret de la liquidation d'une banque s'effectue à l'échelon national ; cela ne peut toutefois s'envisager que sur la base d'une procédure unique s'appliquant à tous les Etats de l'UE. Nous considérons d'un œil critique par contre le projet de la Commission de faire alimenter ce fonds unique par toutes les banques. Les coûts d'une liquidation ne doivent en effet pas être répartis sur l'ensemble des Etats de l'UE, et encore moins imputés à ceux qui présentent un marché bancaire solide. Car il n'y aurait alors qu'un pas, vite franchi, vers un système communautaire unique de garantie des dépôts dans lequel les fonds de garantie prévus à l'origine pour préserver les épargnants par des banques solvables serviraient à renflouer des banques moins vertueuses.

Le groupe des caisses d'épargne allemandes (Sparkassen-Finanzgruppe) estime dès lors que si un tel mécanisme unique de résolution voit le jour, il convient de limiter strictement son intervention aux établissements ayant des activités transfrontières. Et qu'il convient d'insérer celui-ci dans un réseau d'autorités de résolution liées par une étroite coopération. Une telle approche de réseau figure en intégralité dans la directive sur le redressement des banques et la résolution de leurs défaillances, et prévoit, pour le cas de la liquidation de conglomérats d'établissements transfrontières, un mécanisme de coordination entre les autorités nationales concernées sous la forme de la création de comités de liquidation. II convient donc de mettre en œuvre cette directive plutôt que de remettre en question les propositions de règlement avant même leur adoption définitive.

A notre avis, rien ne s'oppose par principe à l'institution, à l'échelon européen et dans le respect des règles de l'Union en matière de résolution, d'une unité centrale de liquidation des groupes d'établissements transfrontières évoqués cidessus. Néanmoins, une mise en œuvre conforme au droit européen présuppose que les procédures administratives de liquidation soient exécutées ou bien par l'intermédiaire des autorités nationales, ou bien que soit adopté un droit communautaire spécifique des procédures administratives et garantissant une protection juridique effective - cela s'impose dans le domaine des liquidations comme dans celui de la surveillance. Dès lors qu'est institué un mécanisme européen de financement, les contributions ne devront être prélevées que sur les groupes d'établissements concernés par le mécanisme de résolution unique. Pour tous les autres établissements, c'est le système des fonds de liquidation nationaux qui devra continuer à s'appliquer.

Lors de la liquidation d'un établissement, il convient d'exclure toute possibilité de lever la garantie des dépôts. En effet, étant donné leur caractère systémique, les banques relevant de la procédure européenne de résolution ne peuvent pas faire l'objet d'une procédure ordinaire de liquidation. Pour ces établissements transfrontières qualifiés naguère de "too-big-to-fail », la procédure doit au contraire être restreinte : lors de l'exécution des mesures, il convient de veiller à ce que les " dépôts garantis » ne puissent en aucune manière être dépréciés (contrairement donc au principe du "bail-in » généralement appliqué). ॥l est au contraire judicieux de mettre à contribution prioritairement, lors de la faillite d'un conglomérat transfrontières, ses actionnaires et autres propriétaires du capital, ainsi qu'également les gros déposants. Cela nous semble mieux correspondre à la finalité d'une telle procédure que de ponctionner indifféremment les contribuables de I'UE. Mais pour les clients d'une banque, cela implique aussi qu'à l'avenir, ils devront se montrer plus prudents encore en choisissant la banque à laquelle ils confient leur épargne ou dans laquelle ils comptent investir. C'est là en toute logique la contrepartie de cette procédure.

En effet, l'organisation des systèmes de garantie des dépôts doit par définition s'effectuer à l'échelon national, c'est-à-dire servir exclusivement la garantie des dépôts des créanciers et ainsi la protection des épargnants. Dans le cas toutefois
... et son intervention devrait se limiter aux établissements transfrontières

Clarifier les procédures de résolution

Pas de bail-in pour la liquidation des banques systémiques

Les systèmes de garantie des dépôts doivent rester à l'échelon national 
Une garantie des dépôts communautarisée détruirait la confiance des déposants

Pour une directive spécifique où un système de garantie des dépôts ne se révélerait plus efficace, on pourrait envisager de faire intervenir subsidiairement le système des différents fonds de restructuration et de liquidation - autrement dit en priorité les banques et non les contribuables.

Nous considérons en effet comme totalement contre-productif et même dommageable un mécanisme de financement contraignant et direct entre les différents systèmes nationaux de garantie des dépôts, voire encore plus une garantie des dépôts européenne communautarisée. Le cas de la Chypre a démontré avec quelle rapidité, y compris au sein d'Etats présentant des structures financières stables, peuvent jaillir des débats sur la sécurité des dépôts, voire naître une vague chaotique de retrait massif des dépôts bancaires (" bank run »). C'est la raison pour laquelle nous nous opposons strictement à une communautarisation de la garantie des dépôts. Car une telle solution européenne saperait, dans les Etats financièrement stables, la confiance des épargnants et, partant, la sécurité de leurs placements. Voilà pourquoi les systèmes de garantie des dépôts doivent rester sous compétence nationale. Cela présente la garantie de prémunir contre les effets de contagion lors des crises bancaires (principe du firewall) et conforte la confiance des déposants dans la fiabilité des systèmes bancaires.

Plutôt que d'envisager une éventuelle communautarisation, il convient à l'inverse de faire aboutir rapidement à l'échelon communautaire les négociations sur la directive relative aux systèmes de garantie des dépôts, bien engagées au sein du trilogue, pour que cette directive soit adoptée par le Conseil et le Parlement européen - sous la forme d'une directive spécifique, et non comme une simple partie de la directive sur le redressement des banques et la résolution de leurs défaillances. Sur les quelques rares points encore en suspens (notamment le montant minimal des garanties de dépôts, l'indemnisation des déposants, la mise en dépôt des fonds de garantie et le calcul des contributions au prorata des risques), un accord doit, et même selon nous peut, être trouvé à brève échéance.

ASSURÉMENT, LE PROJET D'UNION BANCAIRE EUROPÉENNE N'A RIEN D'UN REMÈDE miracle pour résoudre la crise de la finance et de la dette souveraine. A eux seuls, le transfert de compétences et la délégation de la gestion des flux financiers ne suffisent pas à améliorer la qualité de la surveillance des établissements de crédit ni à assurer un changement de comportement face au risque. Cela exige bien plus d'efforts encore, et avant tout la prise de conscience de toutes les parties impliquées du fait que la meilleure prévoyance possible face au risque réside dans des modèles économiques raisonnables qui, non contents de ne pas avoir perdu le contact avec l'économie réelle, placent celle-ci au centre de leurs préoccupations. Dans leurs régions d'implantation, les caisses d'épargne allemandes contribuent étroitement, de par leur modèle économique, au développement des entreprises, essentiellement issues du Mittelstand, et elles ont une connaissance intime de leur clientèle. Certes, nous ne savons pas encore aujourd'hui quels seront les contours de la crise de demain ni quelles en seront les origines. Mais l'expérience nous a prouvé que jamais, jusqu'ici, une politique responsable d'octroi de crédit et un suivi rapproché de la clientèle n'ont eu pour effet de déclencher une crise - bien au contraire, une telle politique s'est toujours révélée un facteur de stabilisation. Voilà un enseignement à ne jamais perdre de vue lorsqu'on cherche à fixer des conditions cadre pour l'activité - de quelque domaine qu'il s'agisse, a fortiori du projet d'une union bancaire.

Traduction : I. Bourgeois

NB : Cette contribution est publiée simultanément par le CIRAC (dans la présente revue), par le Comité d'étude des relations franco-allemandes (Cerfa) auprès de l'Ifri, dans la collection Notes du Cerfa, $\mathrm{n}^{\circ} 103$ (www.ifri.org/?page=nos-e-notes\&id_pub categorie $=7$ ) et, en langue allemande, dans la collection DGAPanalysen de la Deutsche Gesellschaft für Auswärtige Politik (DGAP, Berlin, www.dgap.org). 